\title{
Symbiont acquisition strategy drives host-symbiont associations in the southern Great Barrier Reef
}

\author{
M. Stat $\cdot$ W. K. W. Loh $\cdot$ O. Hoegh-Guldberg \\ D. A. Carter
}

Received: 14 February 2008 / Accepted: 28 July 2008 / Published online: 9 August 2008

(C) Springer-Verlag 2008

\begin{abstract}
Coral larvae acquire populations of the symbiotic dinoflagellate Symbiodinium from the external environment (horizontal acquisition) or inherit their symbionts from the parent colony (maternal or vertical acquisition). The effect of the symbiont acquisition strategy on Symbiodinium-host associations has not been fully resolved. Previous studies have provided mixed results, probably due to factors such as low sample replication of Symbiodinium from a single coral host, biogeographic differences in Symbiodinium diversity, and the presence of some apparently host-specific symbiont lineages in coral with either symbiont acquisition strategies. This study set out to assess the effect of the symbiont acquisition strategy by sampling Symbiodinium from 10 coral species (five with a horizontal and five with a vertical symbiont acquisition strategy) across two adjacent reefs in the southern Great Barrier Reef. Symbiodinium diversity was assessed using singlestranded conformational polymorphism of partial nuclear large subunit rDNA and denaturing gradient gel electrophoresis of the internal transcribed spacer 2 region. The Symbiodinium population in hosts with a vertical symbiont acquisition strategy partitioned according to coral species,
\end{abstract}

Communicated by Biology Editor Dr. Michael Lesser

M. Stat · D. A. Carter

School of Molecular and Microbial Biosciences,

University of Sydney, Sydney, NSW 2006, Australia

M. Stat $(\bowtie)$

Hawaii Institute of Marine Biology, University of Hawaii, P.O. Box 1346, Kaneohe, HI 96744, USA

e-mail: stat@hawaii.edu

W. K. W. Loh · O. Hoegh-Guldberg

The Centre for Marine Studies, University of Queensland,

Brisbane, QLD 4072, Australia while hosts with a horizontal symbiont acquisition strategy shared a common symbiont type across the two reef environments. Comparative analysis of existing data from the southern Great Barrier Reef found that the majority of corals with a vertical symbiont acquisition strategy associated with distinct species- or genus-specific Symbiodinium lineages, but some could also associate with symbiont types that were more commonly found in hosts with a horizontal symbiont acquisition strategy.

Keywords Coral $\cdot$ Dinoflagellate $\cdot$ ITS2 $\cdot$ rDNA . Symbiodinium $\cdot$ Symbiosis

\section{Introduction}

Symbiodinium is a photosynthetic dinoflagellate genus that is found in symbiosis with some protists and a wide range of marine invertebrate hosts, including reef-building corals (Baker 2003). The mutualistic symbiosis between Symbiodinium and corals is considered to be responsible for the growth and formation of coral reefs through the energetic and nutritional advantages of algal-animal endosymbiosis (Muscatine et al. 1981).

There are eight divergent lineages within the genus Symbiodinium, designated clades $\mathrm{A}-\mathrm{H}$, which each contain numerous subclade types based on rDNA (reviewed in Coffroth and Santos 2005; Stat et al. 2006). Patterns of phylogeography are evident for Symbiodinium at the subclade level. Adaptive radiation and limited connectivity have been proposed to explain the large differences in Symbiodinium diversity seen between reefs in the Indo-Pacific compared to the Atlantic-Caribbean (LaJeunesse 2005). On smaller scales, different host-symbiont assemblages can be seen over latitudinal and longitudinal gradients (Baker et al. 
1997; Rodriguez-Lanetty et al. 2001; LaJeunesse et al. 2004a, b; LaJeunesse 2005), and as a function of colony depth (Rowan and Knowlton 1995; Baker and Rowan 1997; Toller et al. 2001; LaJeunesse et al. 2003; IglesiasPrieto et al. 2004) and the irradiance level in individual colonies (Rowan et al. 1997; van Oppen et al. 2001).

Coral hosts acquire Symbiodinium each generation, either by the dinoflagellate symbiont infecting juvenile corals from the ocean environment (horizontal acquisition; Trench 1987) or directly from the parent egg or brooded larvae (maternal or vertical acquisition; Trench 1987). There are conflicting data as to whether this symbiont acquisition strategy influences the final host-symbiont assemblage. Analysis of coral hosts and their associated Symbiodinium genotypes based on the internal transcribed spacer 2 (ITS2) region suggests a correlation between "specialist" Symbiodinium types and hosts with a vertical symbiont acquisition strategy (e.g., LaJeunesse et al. 2003, 2004a, b; LaJeunesse 2005; Thornhill et al. 2006a, b). A high number of unique symbiont types were found in coral hosts from Hawaii (LaJeunesse et al. 2004a), which was considered to be due to the predominance of coral hosts with a maternal symbiont acquisition strategy in Hawaiian reefs. Loh et al. (2001) showed that Seriatopora hystrix (maternal strategist) harbored unique symbiont types in different geographic locations within the Pacific, while Acropora longicyathus (horizontal strategist) had symbionts that were genetically identical between reefs from Australia, Malaysia, and Japan. A study by Barneah et al. (2004) on soft corals from the Red Sea found clade A symbionts associated with hosts that have a vertical symbiont acquisition strategy while clade $\mathrm{C}$ associated with horizontal strategists. In contrast to these observations that largely show symbionts to partition according to host symbiont acquisition strategy, van Oppen (2004) found a similar level of symbiont genetic diversity in Acropora sp. (horizontal strategist) and Montipora sp. (maternal strategist) from the central GBR. Montipora associated with some unique symbiont types inferred to have co-evolved with the host but also harbored symbionts commonly found in Acropora. Other studies employing different coral hosts have found some flexibility among maternal strategists, which sometimes harbor generalist types that are usually confined to symbiosis with horizontal strategists (e.g., Sampayo et al. 2007). In addition, some horizontal strategists can show evidence of specificity, as was observed in Fungia scutaria from Hawaii, which acquires its symbionts from the environment but was found to house the specialist Symbiodinium genotype ITS2 C1f (Weis et al. 2001; Rodriguez-Lanetty et al. 2004).

The difficulty of resolving the effect of symbiont acquisition strategy on Symbiodinium diversity lies in the fact that the above studies did not set out to specifically address this issue or investigate the question using a variety of host species representing both modes of symbiont acquisition. The aim of this study was therefore to systematically sample 10 coral species, five with a horizontal and five with a vertical symbiont acquisition strategy, from two separate locations in the southern Great Barrier Reef, to determine how host symbiont acquisition strategy affects the diversity of their constituent Symbiodinium types.

\section{Materials and methods}

Sample collection

Samples were collected in August 2001 from 10 coral species (14-22 colonies/species, Table 1) at various reef locations surrounding One Tree Island $\left(23^{\circ} 25^{\prime} \mathrm{S} 151^{\circ} 55^{\prime} \mathrm{E}\right)$ and Heron Island $\left(23^{\circ} 25^{\prime} \mathrm{S} 151^{\circ} 55^{\prime} \mathrm{E}\right)$ located in the southern Great Barrier Reef (Fig. 1). Exceptions were colonies of $S$. hystrix collected from location B in January/February 2002 and colonies of Montipora digitata that were collected from location E in July 2002. Coral species that were abundant on these reefs and well characterized with respect to lifecycle were targeted. Conspecific colonies separated by at least $10 \mathrm{~m}$ (to reduce the possibility of collecting the same asexually produced individual) were sampled from intertidal reef flat zones and at depths of $0-4$ and $12-15 \mathrm{~m}$. A $3-\mathrm{cm}$ piece from branching species or a $3-\mathrm{cm}^{2}$ section from massive species was removed using a hammer and chisel. Samples were collected from three sections of each coral colony according to the level of light irradiance (low, intermediate, high) categorized by position on the colony and relative amount of shading. Coral tissue was removed from the skeleton with directed jets of high pressure air supplied using SCUBA, collected into sterile bags, and mixed with an equal amount of saturated salt-DMSO preserving buffer in a microfuge tube for transport and storage (Seutin et al. 1991).

\section{DNA extraction}

Total DNA was extracted using the protocol described in Loh et al. (2001) with minor modifications. Preserved coral/algal tissue was incubated with $1 \% \mathrm{SDS}$ at $65^{\circ} \mathrm{C}$ for $1 \mathrm{~h}$ followed by overnight incubation at $50^{\circ} \mathrm{C}$ with the addition of Proteinase $\mathrm{K}\left(0.5 \mathrm{mg} \mathrm{ml}^{-1}\right)$. Cellular debris was removed by extraction with phenol-chloroform-isoamyl alcohol $(25: 24: 1)$ followed by centrifugation. The supernatant was heated at $65^{\circ} \mathrm{C}$ for 15 min with $1 \% \mathrm{CTAB}$ and recentrifuged. An equal volume of chloroform-isoamyl alcohol (24:1) was then added to the supernatant, which was chilled on ice for $30 \mathrm{~min}$ and centrifuged again. DNA was precipitated from the final supernatant with an equal 
Fig. 1 Location of sites where corals were sampled for Symbiodinium genotyping. (a) The location of sampling sites within the Great Barrier Reef; (b) position of reefs within the Capricorn Bunker Group; (c) Heron Island reef sites $\mathrm{A}$ : intertidal reef flat, B: 0-4 m, C: $12-15 \mathrm{~m}, \mathrm{D}$ : 12-15 m; (d) One Tree reef sites E: 0-4 m, F: 0-4 m, G: 0-4 m, H: $12-15$ m, I: $12-15 \mathrm{~m}$, J: $0-4 \mathrm{~m}$
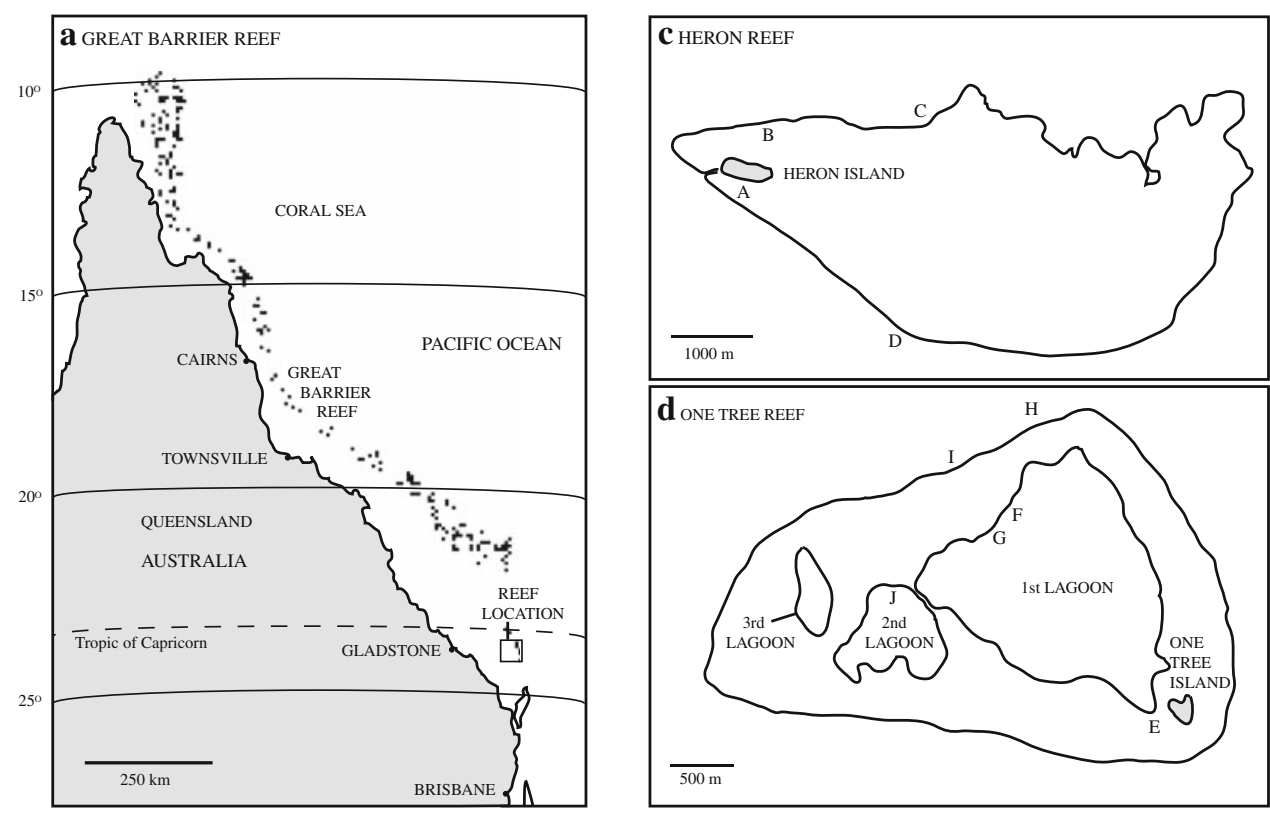

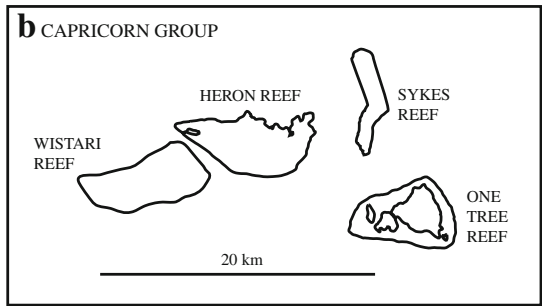

volume of isopropanol and 1/10 volume $3 \mathrm{M}$ sodium acetate ( $\mathrm{pH}$ 5.2). The DNA was washed in $70 \%$ ethanol, dried, and resuspended in TE Buffer $(10 \mathrm{mM}$ Tris-Borate, $1 \mu \mathrm{M}$ EDTA, pH 8.0).

LSU amplification, SSCP, and sequence analysis

Dinoflagellate specific primers were used to amplify the D1/D2 region of the LSU rDNA gene from Symbiodinium (Zardoya et al. 1995; forward: 5' CCC GCT GAA TTT AAG CAT ATA AGT AAG CGG 3' and reverse: 5' GTT AGA CTC CTT GGT CCG TGT TTC AAG A 3'). All PCR reactions contained $10 \mu \mathrm{l} 10 \times \mathrm{PCR}$ buffer $(1 \mathrm{M}$ Tris$\left.\mathrm{HCl}, 0.5 \mathrm{M} \mathrm{KCl}, 2.5 \mathrm{mM} \mathrm{MgCl}_{2}, \mathrm{pH} 8.3\right), 1 \mu \mathrm{l}$ of $5 \mu \mathrm{M}$ each dNTP, $40 \mu \mathrm{g}$ bovine serum albumin, 20 pmol of each primer, and $0.5 \mu \mathrm{l}$ of Taq DNA polymerase $\left(5\right.$ unit $\left.\mu \mathrm{l}^{-1}\right)$ in a $100-\mu l$ reaction. PCR reactions were performed using the following conditions: $94^{\circ} \mathrm{C}$ for $5 \mathrm{~min}$, followed by 30 cycles of $94^{\circ} \mathrm{C}$ for $1 \mathrm{~min}, 65^{\circ} \mathrm{C}$ for $40 \mathrm{~s}$, and $72^{\circ} \mathrm{C}$ for $40 \mathrm{~s}$, and a final extension at $72^{\circ} \mathrm{C}$ for $7 \mathrm{~min}$. Partial LSU rDNA PCR product $(20 \mu \mathrm{l})$ was mixed with an equal volume of stop solution ( $95 \%$ formamide, $10 \mathrm{mM} \mathrm{NaOH}, 0.25 \%$ bromophenol blue, and $0.25 \%$ xylene cyanol), heated at $95^{\circ} \mathrm{C}$ for $8 \mathrm{~min}$ and immediately chilled on ice for $10 \mathrm{~min}$. A $0.5 \times$ MDE vertical gel, prepared according to the manufacturer's instructions (BioWhittaker Molecular Applications,
Rockland, ME, USA), was loaded with the samples and electrophoresed at $140 \mathrm{~V}$ for $14 \mathrm{~h}$ with $0.6 \%$ TBE running buffer (45 mM Tris-Borate, 1 mM EDTA, pH 8.0). The gel was stained in Syber gold (Molecular Probes, Eugene, OR, USA) for $20 \mathrm{~min}$ and viewed under UV transillumination. The image was captured on Polaroid 667 film. Singlestranded conformational polymorphism (SSCP) profiles were compared visually, and variants were selected and electrophoresed together on a single SSCP gel for final comparison. This was done to prevent gel-to-gel variation in SSCP profiles that can occur due to minor differences in gel composition or ambient temperature. All amplifications that gave unique SSCP profiles were directly sequenced. The partial LSU rDNA fragments were purified using GFX $^{\mathrm{TM}}$ PCR DNA purification kits (Amersham Biosciences, New Jersey USA) prior to sequencing on an ABI 3730xl 96-capillary automated DNA sequencer at the Australian Genome Research Facility. DNA sequences were aligned using Clustal W (Thompson et al. 1994) and manually edited using GeneDoc (Nicholas et al. 1997).

\section{ITS2 amplification and DGGE}

ITS2 analysis was undertaken to integrate the data presented in this study with the extensive ITS2 database (e.g., LaJeunesse 2001, 2002, 2005; LaJeunesse et al. 2003, 
2004a, b; Thornhill et al. 2006a, b; Pochon et al. 2007; Sampayo et al. 2007) and to compare the diversity and population structure of Symbiodinium between the two genes. The ITS2 region was amplified as described in LaJeunesse and Trench (2000). Touchdown PCR using the primers ITS2 clamp (5' CGC CCG CCG CGC CCC GCG CCC GTC CCG CCG CCC CCG CCC GGG ATC CAT ATG CTT AAG TTC AGC GGG T 3') and ITSintfor2 (5' GAA TTG CAG AAC TCC GTG $3^{\prime}$ ) was performed starting with an annealing temperature of $62^{\circ} \mathrm{C}$, which was decreased by $0.5^{\circ} \mathrm{C}$ per cycle to $52^{\circ} \mathrm{C}$ and then held for an additional 20 cycles. For denaturing gradient gel electrophoresis (DGGE) analysis, $10 \mu \mathrm{l}$ of ITS2 PCR product was loaded onto an $8 \%$ polyacrylamide denaturing gradient gel (45-80\% urea-formamide gradient; $100 \%$ consists of $7 \mathrm{~mol}^{-1}$ urea and $40 \%$ deionized formamide) and separated by electrophoresis for $9.5 \mathrm{~h}$ at $150 \mathrm{~V}$ at a constant temperature of $60^{\circ} \mathrm{C}$ (C.B.S. Scientific, California USA; LaJeunesse 2002). Profiles were compared to reference samples of known ITS2 types following the protocol of LaJeunesse and Trench (2000), and representative bands of an ITS2 type were confirmed by sequencing following the protocol of LaJeunesse (2002) on a Biodsystems ABI Prism $377^{\mathrm{TM}}$ at the University of Georgia. The gel was stained with Sybr Green (Molecular Probes) for 25 min according to the manufacturer's directions and photographed using 667 Polaroid film.

\section{Statistical analyses}

Analysis of molecular variance (AMOVA) using conventional F-statistics was implemented using the software Arlequin (Excoffier et al. 2005). AMOVA was used to determine the partitioning of SSCP types in (1) hosts with a vertical symbiont acquisition strategy and in (2) hosts with a horizontal symbiont acquisition strategy.

\section{Results}

SSCP and sequence analysis of the partial LSU rDNA region from Symbiodinium

Amplification of the LSU D1/D2 region produced a single fragment of approximately 630 nucleotides from each coral sample. Fifteen different SSCP profiles were identified among the 549 samples (three samples from each of 183 colonies; Fig. 2a). Sequence analysis characterized these as genotypes within Symbiodinium clade C and clade D, and the profiles were designated Clsu1-Clsu 13 and Dlsu1$\mathrm{D} l s u 2$, accordingly. Replicate sequencing was done on SSCP profiles that appeared to be the same but were found in different host colonies or species to confirm that they had the same sequence. This resulted in a total of 23 replicate sequences for Clsu3 (EF372063), 9 for Clsu4 (EF372065), 5 for Clsu5 (EF372066), 6 for Clsu10 (EF372070), and 2 each for Clsu6 (EF372067), Clsu7 (EF372068), and Clsu8 (EF372069). Unambiguous sequence data were obtained from SSCP profiles Clsu4, Clsu5, Clsu7, Clsu8, Clsu10, $\mathrm{D} l s u 1$ (EF372071), and Dlsu2 (EF372072). One sequence originating from the $\mathrm{Clsu3}$ profile amplified from G. favulus differed by a single base change to other sequences derived from this profile, and $\mathrm{Clsu} 6$ contained three polymorphic sites. Unambiguous sequences could not be obtained for Clsu1, Clsu2, Clsu9, Clsu11, Clsu12, and Clsu13, indicating multiple symbiont genotypes or a relatively equal abundance of more than one dominant LSU rDNA sequence within the genome of the symbiont (e.g. Santos et al. 2001; LaJeunesse 2002; Diekmann et al. 2003; van Oppen et al. 2005; Thornhill et al. 2007).

\section{Diversity and distribution of Symbiodinium}

Table 1 shows the Symbiodinium SSCP types associated with each of the coral species at the various different locations, and Fig. 3 represents the SSCP types associated with each host species. Clsu 3 was the most abundant, occurring in 72 different colonies and at all of the reef sites except Heron Island site B. Although Clsu3 occurred in all hosts with a horizontal symbiont transmission strategy, it was not seen in any of the hosts with maternal symbiont transmission. Clsu1, Clsu2, Dlsu1, and Dlsu2 also occurred in hosts with a horizontal symbiont transmission strategy only. Clsu 1 was common to the massive species Favites abdita $(n=3)$, Goniastrea favulus $(n=1)$, and Lobophyllia corymbosa $(n=7)$ and was found in colonies located at all depths and at both reefs. D $l s u 1$ was found in a single colony of G. favulus from the Heron Island reef flat (site A). A single colony of Acropora millepora from One Tree Island reef site $\mathrm{H}$ contained $\mathrm{Clsu} 2$, and two colonies of Acropora palifera at Heron Island reef contained $\mathrm{D} l s u 2$.

Unique SSCP types were found in nearly all of the coral hosts with a maternal transmission strategy. The exception was $\mathrm{Clsu10,}$ which associated with both M. digitata $(n=17)$ and Porites cylindrica $(n=17) . C l s u 10$ was the only symbiont type found in these host species, apart from a single colony of M. digitata that contained both Clsu10 and Clsu 11 (see below). All colonies of S. hystrix $(n=20)$ harbored Clsu9. Pocillopora damicornis colonies present at all depths harbored Clsu12 $(n=14)$, and six colonies at 12 $15 \mathrm{~m}$ contained Clsu13. Stylophora pistillata associated with five SSCP types: Clsu4 $(n=10), \mathrm{Clsu} 5(n=5), \mathrm{Clsu6}$ $(n=2), \mathrm{Clsu} 7(n=1)$, and Clsu8 $(n=1)$. Clsu4 was present in colonies located at depths of $0-4$ and $12-15 \mathrm{~m}$ at both reefs. Clsu 5 was only found in colonies on the reef flat at Heron Island. Clsu6 occurred in two colonies in shallow 


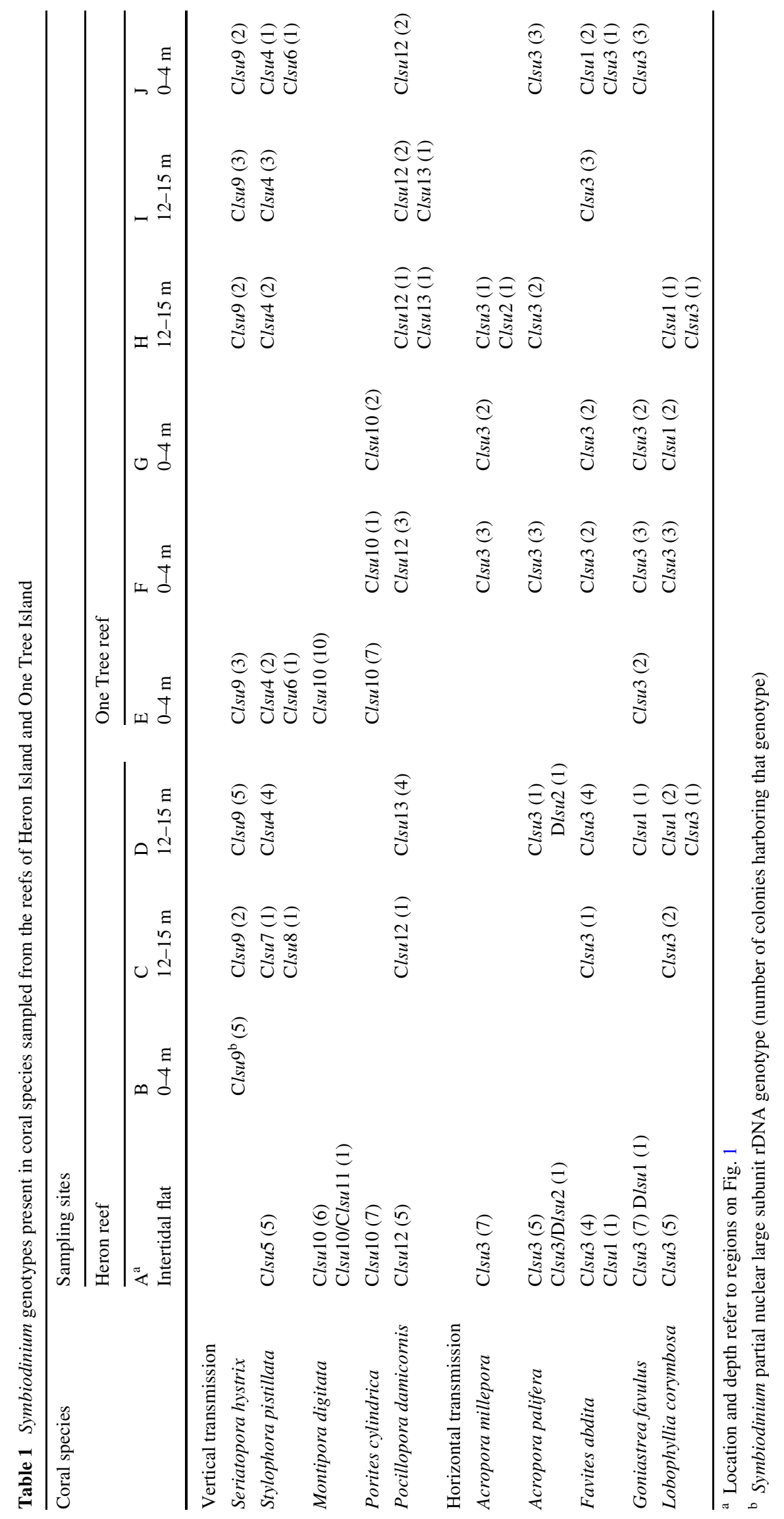




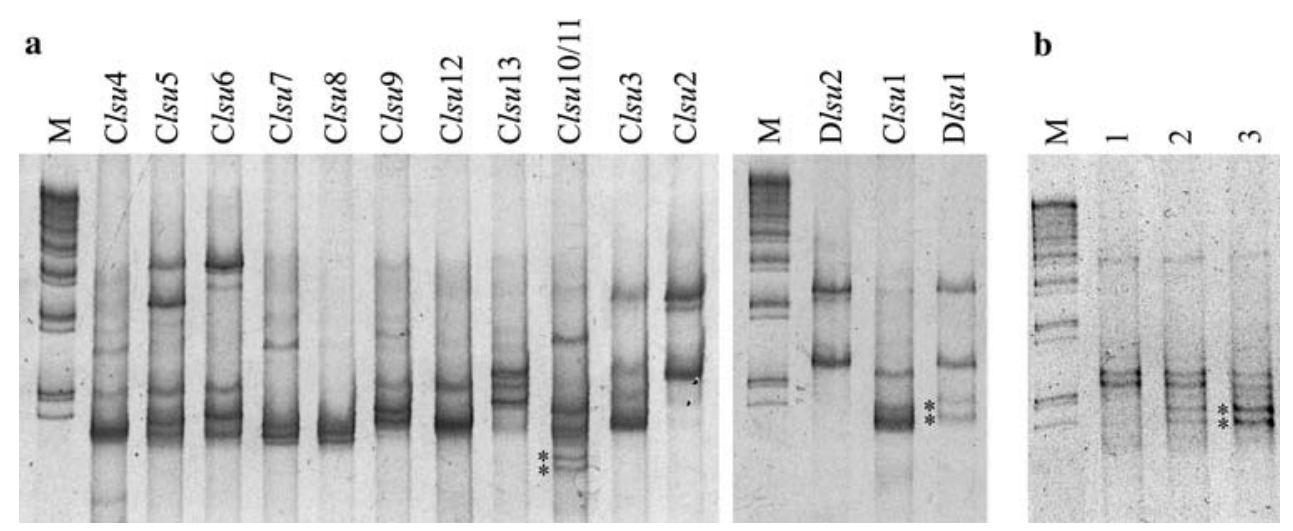

Fig. 2 Single-stranded conformational polymorphism gel showing Symbiodinium partial nuclear large subunit rDNA fingerprints. Clsu1Clsu 13 and Dlsu1-Dlsu2 represent Symbiodinium genotypes within the phylogenetic groups clades $\mathrm{C}$ and $\mathrm{D}$, respectively (a). Clsu11 is distinguished from $\mathrm{Clsu} 10$ by two small bands, each indicated by an asterisk. Similarly, D $l s u 1$ and $\mathrm{D} l s u 2$ are distinguished by two small bands, each indicated by an asterisk. SSCP profiles Clsu 10 and Clsu 11 originating from the same colony of Montipora digitata sampled at an area of high (1), intermediate (2), and low (3) irradiance (b). Asterisks indicate bands associated with SSCP type Clsu11. M: marker
Fig. 3 Symbiodinium genotypes associated with each of the coral hosts sampled in the study based on LSU rDNA analysis. Each segment within the pie chart represents one coral. Multi-colored pie segments indicate a colony that associated with two SSCP rDNA

Symbiodinium types: Clsu10/11 in Montipora digitata and $\mathrm{Clsu3/}$ $\mathrm{D} l s u 2$ in Acropora palifera

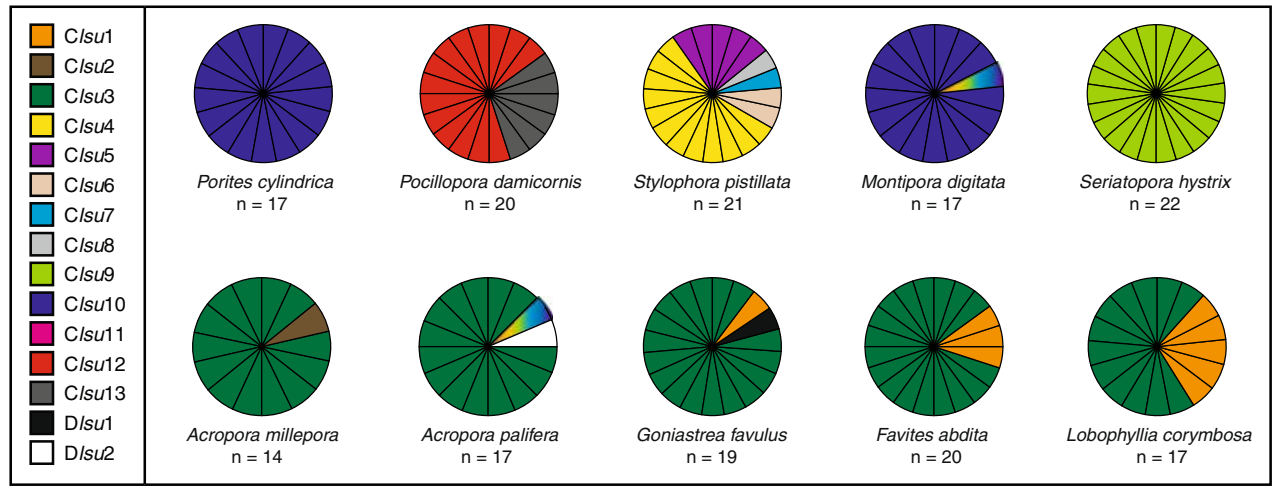

using DGGE of the ITS2 region included Clus1 (from G. favulus: $n=1$ and L. corymbosa: $n=1$ ), Clsu3 (from A. millepora: $n=13$, A. palifera: $n=15, F$. abdita: $n=3$, G. favulus: $n=2$, and L. corymbosa: $n=3$ ), Clsu4 (from S. pistillata: $n=3$ ), Clsu5 (from S. pistillata: $n=3$ ), Clsu6 (from S. pistillata: $n=2$ ), Clsu7 (from S. pistillata: $n=1$ ), Clsu8 (from S. pistillata; $n=1$ ), Clsu9 (from S. hytrix: $n=13$ ), Clsu10 (from M. digitata: $n=14$, and P. cylindrica: $n=17$ ), Clsu11 (from M. digitata: $n=1$ ), Clsu12 (from P.damicornis: $n=3$ ), Clsu13 (from $P$. damicornis: $n=3$ ), Dlsu1 (from G. favulus: $n=1$ ), and Dlsu2 (from A. palifera: $n=2$ ). DGGE profiles correlated with SSCP profiles so that a single DGGE profile was observed for all samples producing a given SSCP profile. Exceptions were Clsu12 which produced the ITS2-DGGE patterns representing $\mathrm{C} 42 / \mathrm{C} 42 \mathrm{a}$ and $\mathrm{C} 42 / \mathrm{C} 42 \mathrm{a} / \mathrm{C} 42 \mathrm{~b}$, and $\mathrm{Clsu} 5$ and $\mathrm{Clsu} 6$ which both gave ITS2 pattern C78.

\section{Community structure of Symbiodinium}

Partitioning of LSU SSCP types according to host symbiont acquisition strategy was found to be highly significant 
Fig. 4 Internal transcribed sequence 2 (ITS2) DGGE profiles of Symbiodinium identified in the coral hosts sampled in the study. The corresponding LSU rDNA type is indicated at the top of each lane. M: ITS2-DGGE standards

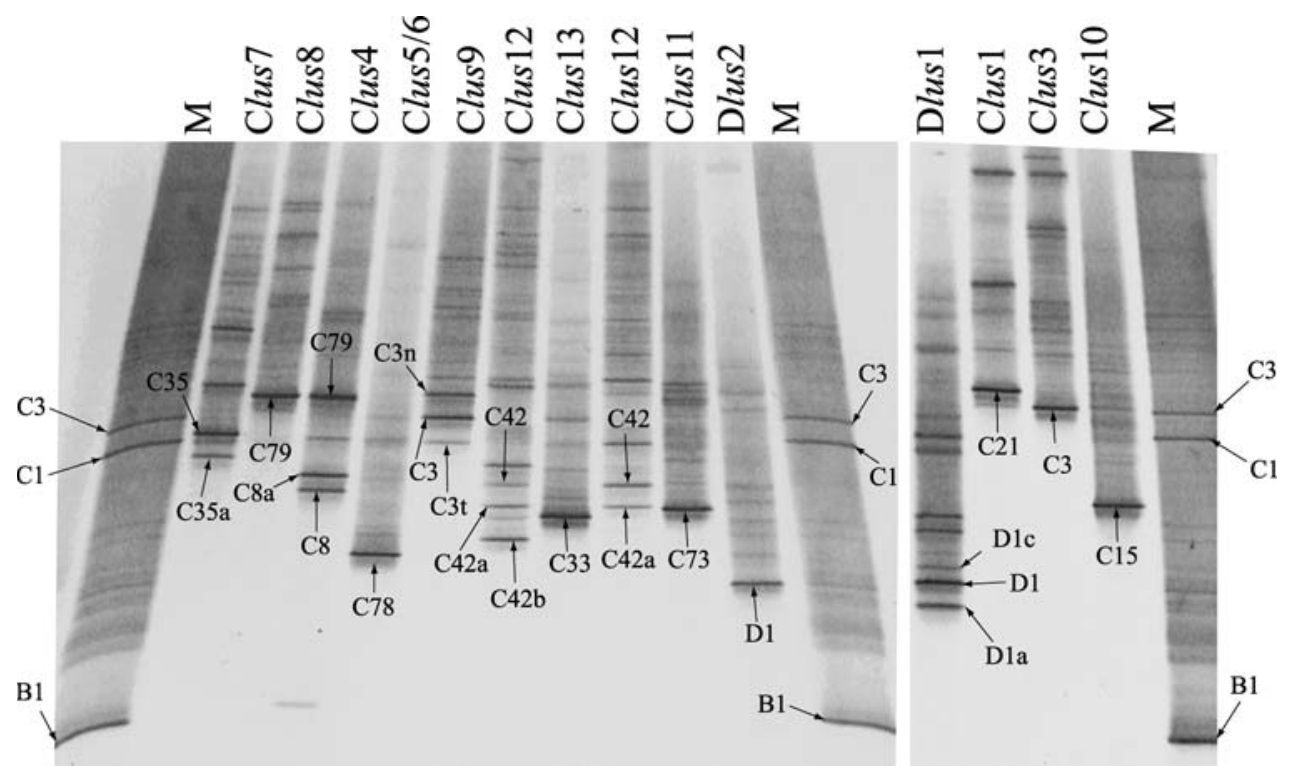

Table 2 Analysis of molecular variance of hosts within each symbiont acquisition strategy

\begin{tabular}{|c|c|c|c|c|c|c|}
\hline Source & df & SS & Var comp & $\operatorname{Var}(\%)$ & $\begin{array}{l}\phi C T \\
\phi S C\end{array}$ & Sig \\
\hline \multicolumn{7}{|c|}{ Vertical acquisition } \\
\hline Hosts & 4 & 26.814 & 0.331 & 71.23 & 0.712 & 0.002 \\
\hline Reef (host) & 5 & 1.472 & 0.019 & 4.03 & 0.140 & 0.036 \\
\hline Within reef & 87 & 10.003 & 0.115 & 24.74 & & \\
\hline Total & 96 & 38.289 & 0.465 & 100.00 & & \\
\hline \multicolumn{7}{|c|}{ Horizontal acquisition } \\
\hline Hosts & 4 & 0.914 & 0.005 & 3.59 & 0.036 & 0.188 \\
\hline Reef (host) & 5 & 0.721 & 0.002 & 1.35 & 0.014 & 0.308 \\
\hline Within reef & 77 & 9.894 & 0.128 & 95.06 & & \\
\hline Total & 86 & 11.528 & 0.135 & & & \\
\hline
\end{tabular}

The hosts for vertical acquisition are Seriatopora hystrix, Stylophora pistillata, Montipora digitata, Porites cylindrica, and Pocillopora damicornis and for horizontal acquisition are Acropora millepora, Acropora palifera, Favites abdita, Goniastrea favulus, and Lobophyllia corymbosa. The regional variance relative to the total variance is $\phi_{\mathrm{CT}}$. The between site within host variance divided by the sum of itself and the within site variance is $\phi_{\mathrm{SC}}$. Significant values $(\alpha=0.05)$ are indicated in bold font

(Likelihood ratio test; 255.81, $\mathrm{df}=14, P<0.0001$ ). There was a significant association between coral species with a vertical symbiont acquisition strategy and the associated symbiont SSCP type (Likelihood ratio test; 267.43, df $=36$, $P<0.0001)$ indicating host-symbiont specificity within this group of corals. In contrast, there was no significant association between coral species with a horizontal symbiont acquisition strategy and the associated symbiont SSCP type, indicating a shared symbiont type for this group of corals.

Symbiodinium SSCP types were grouped by host and reef for AMOVA (Table 2). Two independent AMOVAs were performed: (1) for hosts with a vertical symbiont acquisition strategy and (2) for hosts with a horizontal symbiont acquisition strategy. In hosts with a vertical symbiont acquisition strategy, there was a significant effect of host on the community of Symbiodinium $\left(\phi_{\mathrm{CT}}=0.712, P=0.002\right.$, Table 2) and a significant effect of reef nested within host ( $\phi_{\mathrm{SC}}=0.140, P=0.036$, Table 2$)$. In contrast, for coral with a horizontal symbiont acquisition strategy, partitioning of Symbiodinium due to host or by reef nested within host was not significant (Table 2).

\section{Discussion}

This study supports the hypothesis that the mechanism by which corals obtain their symbionts strongly influences host-symbiont assemblages. Symbiodinium genotypes from corals with a maternal symbiont acquisition strategy were 
largely host-specific, while all corals included with horizontal symbiont acquisition strategies shared a lower diversity of symbiont types.

Although hosts employing a maternal symbiont acquisition strategy all harbored distinct Symbiodinium genotypes, differences in specificity and diversity were evident within this group of corals. M. digitata and P. cylindrica both harbored almost exclusively Clsu10 (ITS2 C15), a symbiont specific to these corals and found in several other species of Porites and the hydroid Aglaophenia sp. in the region (this study; LaJeunesse et al. 2003). M. digitata also harbored Clsu11 (ITS2 C73), which has not been found in any other coral species (LaJeunesse 2005). S. hystrix, harbored Clsu 9 (ITS2 C3n-t) exclusively, although it has also been shown to associate with the global generalist symbiont ITS2 C3 when sampled in this region (LaJeunesse et al. 2003; Sampayo et al. 2007). Numerous Symbiodinium types were found in S. pistillata and P. damicornis. S. pistillata harbored symbiont types Clsu4 (ITS2 C8/C8a), Clsu5 (ITS2 C78), Clsu6 (ITS2 C78), Clsu7 (ITS2 C35/C35a), and Clsu8 (ITS2 C79). In addition to these, the global generalist symbiont ITS2 $\mathrm{C} 1$ has been detected in colonies of S. pistillata (LaJeunesse et al. 2003; Sampayo et al. 2007). Similarly, in addition to symbiont types Clsu 12 (ITS2 C42/ $\mathrm{C} 42 \mathrm{a} / \mathrm{C} 42 \mathrm{~b}$ ) and $\mathrm{Clsu} 13$ (ITS2 C33) found in $P$. damicornis, the global generalist $\mathrm{C} 1$ and other symbionts not specific to $P$. damicornis (ITS2 $\mathrm{C} 1 \mathrm{~b}$ and $\mathrm{C} 1 \mathrm{c}$ ) have been found in this vertical strategist (LaJeunesse et al. 2003; Sampayo et al. 2007). A faint band at the migration endpoint for ITS2 C1 in our DGGE profiles for Clsu12/13 also points to the presence of $\mathrm{C} 1$ in this host species. Collectively, these findings show that host-specific symbiont lineages predominate in coral with a vertical symbiont acquisition strategy in the southern Great Barrier Reef and that generalist symbionts can also associate with these corals, as has been suggested by van Oppen (2004). ITS2 types $\mathrm{C} 3$ and $\mathrm{C} 1$ have been called the "living ancestors" within the Symbiodinium clade C lineage (LaJeunesse 2005). Thus, even though host-symbiont specificity has evolved in coral lineages with a vertical symbiont acquisition strategy, the recognition between these ancestral types (from which the specialist symbionts are hypothesized to have evolved) and this group of corals appears to remain a viable interaction.

Genotypes Clsu 1 (ITS2 C21) and Clsu3 (ITS2 C3) were common to hosts with horizontal symbiont acquisition strategies and were truly ubiquitous, being present in corals at both study sites and at various depths. It is likely that retaining the ability to maintain symbiotic interactions with a wide range of host species and to persist when outside the host has restricted the genetic differentiation, and hence speciation of these horizontally transmitted symbionts. Although host-specific symbiont lineages were not detected in this group of corals in the current study, specificity between the horizontal strategist $F$. scutaria and the specialist genotype ITS2 C1f has been shown in coral originating from Hawaii (Weis et al. 2001; Rodriguez-Lanetty et al. 2004). This suggests that specific host-symbiont assemblages can also occur in corals that acquire their symbionts from the environment so that a particular symbiont is selected over the generalist type (e.g., C1 and/or C3), but this is apparently a rare scenario.

Spatial distribution of different symbiont types within a coral colony and with colony depth was rare. A single colony of A.palifera harbored both Clsu3 (ITS2 C3) and Dlsu2 (ITS2 D1) and one colony of $M$. digitata harbored Clsu 10 (ITS2 C15) and Clsu11 (ITS2 C73), with the latter appearing to be distributed over the colony according to relative light levels. The ability to accommodate symbionts that are genetically variable may provide the host with benefit over a wider environmental range (Douglas 1998). However, $P$. damicornis and S. pistillata appear to be more susceptible to bleaching than $M$. digitata and P. cylindrica, which associate with a single Symbiodinium genotype but are relatively bleach resistant (Loya et al. 2001; LaJeunesse et al. 2003). Recently, Mieog et al. (2007) found colonies of Acropora contained multiple symbiont types that could not be detected using standard PCR and fingerprinting techniques. It is possible that this finding may extend to many more host taxa. These cryptic symbiont populations may be kept at low levels, as mixed Symbiodinium communities have been shown to be less beneficial to the host animal (Loram et al. 2007).

The LSU rDNA and ITS2 regions are linked in the rDNA array (Long and Dawid 1980). ITS2 is under less functional constraint than the LSU region and is therefore likely to be more variable (LaJeunesse 2001). The level of Symbiodinium diversity resolved in this study using these two markers was similar, and the few exceptions could be the result of primer bias, or because different techniques were used to analyze the diversity of the amplified fragments. The difference in Symbiodinium diversity resolved in this study compared to others performed in the Southern Great Barrier Reef, and particularly the lack of generalist types seen among the vertical strategists in our analysis compared to others (LaJeunesse et al. 2003; Sampayo et al. 2007) is likely due to differences in the number of colonies sampled in the study (where increased sampling can reveal rare host-symbiont partnerships; e.g., Sampayo et al. 2007), the site at which the colony was collected (fine-scale niche partitioning; e.g., Sampayo et al. (2007)), or the multi-copy nature of rDNA and interpretation of DGGE gels. For example, the ITS2-DGGE profile for Clsu 9 contained bands C3, C3n, and C3t. Are these all from the same genome or do they represent different symbiont types? In addition, some bands were fainter than others within a 
single profile, such as the $\mathrm{C} 1$ band present in the $\mathrm{Clsu12/13}$ samples. Researchers may differ in how they interpret the significance of these minor bands and whether they warrant inclusion in the analysis, which affects the number of symbiont types inferred to be present in a sample. The challenges presented by the multi-copy nature of the ITS2 gene and using DGGE to interpret Symbiodinium diversity have previously been recognized (Apprill and Gates 2007; Thornhill et al. 2007) and argue for the development of new approaches such as multilocus sequence typing (MLST; e.g., Adiri et al. 2003), based on a suite of single copy loci, to characterize functional groups of Symbiodinium.

Mutualisms employing vertical transmission strategies may be more beneficial given that a host with a horizontal acquisition system may fail to acquire symbionts (GenkaiKato and Yamamura 1999). Indeed, the evolution of the mutualistic symbiosis between macrotermitine termites and their ectosymbiotic fungi reveals vertical transmission as the derived transmission strategy (Aanen et al. 2002). The localization of Symbiodinium during the development from the host egg to mature planulae is different between species, suggesting that vertical symbiont acquisition has evolved multiple times (Hirose and Hidaka 2006). If vertical symbiont acquisition is a more risk-free approach to acquiring symbionts, then why do the majority of coral species possess a horizontal mode of symbiont acquisition? One argument is that horizontal symbiont acquisition allows plasticity in the symbiosis, where hosts may acquire different genetic varieties of symbionts and occupy numerous environmental niches or adapt with environmental change (Buddemeier and Fautin 1993; Douglas 1998). The ability to "switch" symbionts has been proposed (Buddemeier and Fautin 1993; Baker 2001; Baker et al. 2004), but evidence in support of the acquisition of completely new symbiont types in natural coral ecosystems over ecological time scales is currently absent (Hoegh-Guldberg et al. 2002; Stat et al. 2006). If acquisition of exogenous symbionts can occur in adult colonies, the data presented here indicate acquisition of generalist types may be possible for most coral species, but specialist symbionts (which make up the majority of diversity within Symbiodinium) are restricted to their coral lineage. Ultimately, questions on the degree of host-symbiont flexibility and the adaptive potential of coral will become increasingly important, as we strive to understand and project the changes of coral reefs to global climate change.

Acknowledgments The authors would like to thank T. Bui, K. Low, X. Pochon, T. LaJeunesse, W. Fitt, G. Schmidt, R. Gates, the staff at One Tree and Heron Island, and the reviewers who helped to improve this manuscript. This research was supported by an Australian Postgraduate Award to M. Stat and funded by an Australian Research Council grant (A10009205) to O. Hoegh-Guldberg and D. Carter, an Australian Biological Resources Study grant 204-53 to D. Carter, and by the GEF-World Bank Coral Reef Targeted Research Project (www.gefcoral.org).

\section{References}

Aanen DK, Eggleton P, Rouland-Lefevre C, Guldberg-Froslev T, Rosendahl S, Boomsma JJ (2002) The evolution of fungus-growing termites and their mutualistic fungal symbionts. Proc Natl Acad Sci USA 99:14887-14892

Adiri RS, Gophna U, Ron EZ (2003) Multilocus sequence typing (MLST) of Escherichia coli 078 strains. FEMS Microbiol Lett 222:199-203

Apprill AM, Gates RD (2007) Recognizing the diversity in coral symbiotic dinoflagellate communities. Mol Ecol 16:1127-1134

Baker AC (2001) Reef corals bleach to survive change. Nature 411:765-766

Baker A (2003) Flexibility and specificity in coral-algal symbiosis: diversity, ecology, and biogeography. Annual Review of Ecology, Evolution, and Systematics 34:661-689

Baker AC, Rowan R (1997) Diversity of symbiotic dinoflagellates (zooxanthellae) in scleractinian corals of the Caribbean and Eastern Pacific. Proc 8th Intl Coral Reef Symp 2:1301-1306

Baker AC, Rowan R, Knowlton N (1997) Symbiosis ecology of two Caribbean Acroporid corals. Proc 8th Intl Coral Reef Symp 2:1295-1300

Baker AC, Starger CJ, McClanahan TR, Glynn PW (2004) Corals' adaptive response to climate change. Nature 430:741

Barneah O, Weis VM, Perez S, Benayahu Y (2004) Diversity of dinoflagellate symbionts in Red Sea soft corals: mode of symbiont acquisition matters. Mar Ecol Prog Ser 275:89-95

Buddemeier R, Fautin D (1993) Coral bleaching as an adaptive mechanism. Bioscience 43:320-325

Coffroth MA, Santos SR (2005) Genetic diversity of symbiotic dinoflagellates in the genus Symbiodinium. Protist 156:19-34

Diekmann OE, Olsen JL, Stam WT, Bak RPM (2003) Genetic variation within Symbiodinium clade B from the coral genus Madracis in the Caribbean (Netherlands Antilles). Coral Reefs 22:29-33

Douglas A (1998) Host benefit and the evolution of specialization in symbiosis. Heredity 81:599-603

Excoffier L, Laval G, Schneider S (2005) Arlequin ver 3.0: an integrated software package for population genetic data analysis. Evol Bioinformatics Online 1:47-50

Genkai-Kato M, Yamamura N (1999) Evolution of mutualistic symbiosis without vertical transmission. Theor Popul Biol 5:309-323

Hirose M, Hidaka M (2006) Early development of zooxanthella-containing eggs of the corals Porites cylindrica and Montipora digitata: the endodermal localization of zooxanthellae. Zool Sci 23:873-881

Hoegh-Guldberg O, Jones RJ, Ward S, Loh WK (2002) Is coral bleaching really adaptive? Nature 415:601-602

Iglesias-Prieto R, Beltran VH, LaJeunesse TC, Reyes-Bonilla H, Thome PE (2004) Different algal symbionts explain the vertical distribution of dominant reef corals in the eastern Pacific. Proc R Soc Lond, B 271:1757-1763

LaJeunesse TC (2001) Investigating the biodiversity, ecology, and phylogeny of endosymbiotic dinoflagellates in the genus Symbiodinium using the ITS region: in search of a "species" level marker. J Phycol 37:866-880

LaJeunesse TC (2002) Diversity and community structure of symbiotic dinoflagellates from Caribbean coral reefs. Mar Biol 141:387400

LaJeunesse TC (2005) "Species" radiations of symbiotic dinoflagellates in the Atlantic and Indo-Pacific since the Miocene-Pliocene Transition. Mol Biol Evol 22:570-581 
LaJeunesse TC, Trench RK (2000) Biogeography of two species of Symbiodinium (Freudenthal) inhabiting the intertidal sea anemone Anthopleura elegantissima (Brandt). Biol Bull 199:126-134

LaJeunesse TC, Loh WKW, van Woesik R, Hoegh-Guldberg O, Schmidt GW, Fitt WK (2003) Low symbiont diversity in southern Great Barrier Reef corals, relative to those of the Caribbean. Limnol Oceanogr 48:2046-2054

LaJeunesse TC, Thornhill DJ, Cox EF, Stanton FG, Fitt WK, Schmidt GW (2004a) High diversity and host specificity observed among symbiotic dinoflagellates in reef communities from Hawaii. Coral Reefs 23:596-603

LaJeunesse TC, Bhagooli R, Hidaka M, de Vantier L, Done T, Schmidt GW, Fitt WK, Hoegh-Guldberg O (2004b) Closely related Symbiodinium spp. differ in relative dominance in coral reef host communities across environmental, latitudinal and biogeographic gradients. Mar Ecol Prog Ser 284:147-161

Loh WKW, Loi T, Carter D, Hoegh-Guldberg O (2001) Genetic variability of the symbiotic dinoflagellates from the wide ranging coral species Seriatopora hystrix and Acropora longicyathus in the Indo-West Pacific. Mar Ecol Prog Ser 222:97-107

Long EO, Dawid IB (1980) Repeated genes in eukaryotes. Annu Rev Biochem 49:727-764

Loram JE, Trapido-Rosenthal H, Douglas AE (2007) Functional significance of genetically different symbiotic algae Symbiodinium in a coral reef symbiosis. Mol Ecol 16:4849-4857

Loya Y, Sakai K, Yamazato K, Nakano Y, Sambali H, van Woesik R (2001) Coral bleaching: the winners and the losers. Ecol Lett 4:122-131

Mieog JC, van Oppen JH, Cantin NE, Stam WT, Olsen JL (2007) Realtime PCR reveals a high incidence of Symbiodinium clade D at low levels in four scleractinian corals across the Great Barrier Reef: implications for symbiont shuffling. Coral Reefs 26:449-457

Muscatine L, McCloskey LR, Marian RE (1981) Estimating the daily contribution of carbon from zooxanthellae to coral animal respiration. Limnol Oceanogr 26:601-611

Nicholas KB, Nicholas HB Jr, Deerfield DW II (1997) GeneDoc: analysis and visualization of genetic variation. EMBNEW.NEWS 4:14, http://www.nrbsc.org/downloads/

Pochon X, Garcia-Cuetos L, Baker AC, Castella E, Pawlowski J (2007) One-year survey of a single Micronesian reef reveals extraordinarily rich diversity of Symbiodinium types in soritid foramnifera. Coral Reefs 26:867-882

Rodriguez-Lanetty M, Loh W, Carter D, Hoegh-Guldberg O (2001) Latitudinal variability in symbiont specificity within the widespread scleractinian coral Plesiastrea versipora. Mar Biol 138:1175-1181

Rodriguez-Lanetty M, Krupp DA, Weis VM (2004) Distinct ITS2 types of Symbiodinium in clade C correlate with cnidarian/dinoflagellate specificity during onset of symbiosis. Mar Ecol Prog Ser 275:97-102

Rowan R, Knowlton N (1995) Intraspecific diversity and ecological zonation in coral-algal symbiosis. Proc Natl Acad Sci USA 92:2850-2853
Rowan R, Knowlton N, Baker A, Jara J (1997) Landscape ecology of algal symbionts creates variation in episodes of coral bleaching. Nature 388:265-269

Sampayo EM, Franceschinis L, Hoegh-Guldberg O, Dove S (2007) Niche partitioning of closely related symbiotic dinoflagellates. Mol Ecol 16:3721-3733

Santos SR, Taylor DJ, Coffroth MA (2001) Genetic comparisons of freshly isolated versus cultured symbiotic dinoflagellates: Implications for extrapolating to the intact symbiosis. J Phycol 37:900-912

Seutin G, White BN, Boag PT (1991) Preservation of avian blood and tissue samples for DNA analyses. Can J Zool 69:82-90

Stat M, Carter D, Hoegh-Guldberg O (2006) The evolutionary history of Symbiodinium and scleractinian hosts - symbiosis, diversity, and the effect of climate change. Perspect Plant Ecol Evol Syst $8: 23-43$

Thompson JD, Higgins DG, Gibson TJ (1994) ClustalW: Improving the sensitivity of progressive multiple sequence alignment through sequence weighting, position-specific gap penalties and weight matrix code. Nucleic Acids Res 22:4673-80

Thornhill DJ, Fitt WK, Schmidt GW (2006a) Highly stable symbiosis among western Atlantic brooding corals. Coral Reefs 25:515-519

Thornhill DJ, LaJeunesse TC, Kemp DW, Fitt WK, Schmidt GW (2006b) Multi-year, seasonal genotypic surveys of coral-algal symbioses reveal prevalent stability of post-bleaching reversion. Mar Biol 148:711-722

Thornhill DJ, LaJeunesse TC, Santos SR (2007) Measuring rDNA diversity on eukaryotic microbial systems: how intragenomic variation, pseudogenes, and PCR artifacts confound biodiversity estimates. Mol Ecol 16:5326-5340

Toller WW, Rowan R, Knowlton N (2001) Zooxanthellae of the Montastraea annularis species complex: patterns of distribution of four taxa of Symbiodinium on different reefs and across depths. Biol Bull 201:348-359

Trench RK (1987) Dinoflagellates in non-parasitic symbioses. In: Taylor F (ed) The biology of dinoflagellates. Blackwell, Oxford, pp 530-570

van Oppen MJH (2004) Mode of zooxanthella transmission does not affect zooxanthella diversity in acroporid corals. Mar Biol 144:1-7

van Oppen MJH, Palstra FP, Piquet AM-T, Miller DJ (2001) Patterns of coral-dinoflagellate associations in Acropora: significance of local availability and physiology of Symbiodinium strains and host-symbiont selectivity. Proc R Soc Lond, B 268:1759-1767

van Oppen MJH, Mieog JC, Sanchez CA, Fabricius KE (2005) Diversity of algal endosymbionts (zooxanthellae) in octocorals: the roles of geography and host relationships. Mol Ecol 14:2403-2417

Weis VM, Reynolds WS, deBoer MD, Krupp DA (2001) Host-symbiont specificity during onset of symbiosis between the dinoflagellates Symbiodinium spp. and planula larvae of the scleractinian coral Fungia scutaria. Coral Reefs 20:301-308

Zardoya R, Costas E, Lopez-Rodas V, Garrido-Pertierra A, Bautista JM (1995) Revised dinoflagellate phylogeny inferred from molecular analysis of large-subunit ribosomal RNA gene sequences. J Mol Evol 51:26-40 